\title{
AGROQUÍMICOS Y SUFRIMIENTO AMBIENTAL: REFLEXIONES DESDE LAS CIENCIAS SOCIALES
}

\section{PESTICIDES AND ENVIRONMENTAL SUFFERING: PERSPECTIVES FROM THE SOCIAL SCIENCES}

\author{
Sindy Mora Solano ${ }^{1}$ \\ sindymora@gmail.com \\ sindy.mora@ucr.ac.cr
}

\begin{abstract}
Resumen
En este artículo se exponen los principales temas discutidos en el marco de las Jornadas de Reflexión de Ciencias Sociales, en donde fueron discutidos algunos resultados de una investigación en torno al movimiento de los trabajadores bananeros afectados por el nemagón (DBCP), investigación que se realiza desde el Instituto de Investigaciones Sociales (IIS). Cuáles pueden ser los aportes de las ciencias sociales en temas relacionados con el uso y exposición a los agroquímicos es el objeto discutido en este articulo.

Palabras clave: Monocultivos, agroquímicos, nemagón, DBCP, movimientos sociales, organización politica, salud, ciencias sociales.
\end{abstract}

\begin{abstract}
In this paper I expose some issues that were discussed in the Jornadas de Reflexion de Ciencias Sociales. In this activity were exposed some results from a research about the study case of former banana workers who have been affected by the use of Nemagon (DBCP). The article suggests some analytical perspectives to recognize the contributions from social science to understand the use and consequences of pesticides.
\end{abstract}

Keywords: Monoculture, pesticides, nemagón, DBCP, social movements, political organization, health, social science.

1 Investigadora e integrante del Programa Nuevas Formas de Acumulación, Distribución y Desigualdad (PADD), del Instituto de Investigaciones Sociales, Universidad de Costa Rica, Sede Rodrigo Facio 


\section{Introducción}

Las reflexiones presentadas seguidamente forman parte de un proyecto de investigación titulado "Las consecuencias del "oro verde": el movimiento de las y los trabajadores bananeros afectados por el nemagón en Costa Rica entre 1990 y 2010". Este proyecto se encuentra inscrito en el Programa Nuevas Formas de Acumulación, Distribución y Desigualdad (PADD), del Instituto de Investigaciones Sociales (IIS).

Una de las preguntas que pueden plantearse en el marco de unas jornadas de reflexión en torno al quehacer de las ciencias sociales, es cuál es la relación que existe entre el uso de los agroquímicos y las disciplinas de las ciencias sociales. De la misma manera, es posible también preguntarse si éste no es un tema que debe ser abordado por parte de especialistas en sustancias tóxicas o por parte de los profesionales del área de la salud.

A pesar de la formulación de estas preguntas, la experiencia de investigación realizada desde el Instituto de Investigaciones Sociales (IIS) muestra la importancia de entender el uso y la exposición a los agroquímicos desde la perspectiva de estas disciplinas, entendiendo además, las implicaciones que estos procesos han tenido en nuestro país. Para ello, es central considerar la expansión de los monocultivos desarrollados en las distintas regiones, la dependencia a los agroquímicos en el marco de la producción de los monocultivos, la exposición a estas sustancias, no sólo por parte de la población trabajadora, sino también, por parte de las poblaciones y comunidades cercanas, así como, los efectos en la salud, los efectos sociales, psicológicos y los efectos políticos que pueden tener estas dinámicas.

En el presente texto se exponen los principales temas discutidos al respecto, en el marco de las Jornadas de Reflexión de Ciencias Sociales, jornadas realizadas en el mes de mayo del año 2013, y organizadas por la Facultad de Ciencias Sociales de la Universidad de Costa Rica.

\section{El nemagón, el sufrimiento ambiental y las posibles miradas desde las ciencias sociales}

El DiBromoCloroPropano o DBCP, agroquímico conocido con el nombre de nemagón, fue la sustancia utilizada entre los años 60 y 70 en las zonas bananeras para combatir los nematodos, una especie de gusanos que se encontraba atacando la producción del banano (Giralt, 1996; Bohme, 2012; Barraza; Jansen; van Wendel; Wesswling, 2013).

La aplicación manual de este agroquímico, durante el periodo mencionado, produjo una serie de consecuencias en la salud y en la vida social, familiar y en las subjetividades de las trabajadoras y los trabajadores bananeros quienes se vieron afectados por dicha sustancia. Si bien se ha reconocido la esterilidad masculina como la principal secuela del uso y la exposición al nemagón, el trabajo de campo realizado en el marco del proyecto Las consecuencias del "oro verde", ha mostrado una serie de secuelas producto del uso y la exposición al agroquímico, que trascienden dicha esterilidad. Afectaciones en la piel, en las articulaciones, problemas de la vista, dolores de cabeza, abortos en las mujeres, pero también, cambios en las relaciones familiares, rupturas en las relaciones de pareja, dificultades para establecer relaciones de pareja, producto fundamentalmente de la esterilidad masculina, y procesos de estigmatización hacia los hombres afectados por el nemagón, son algunas de estas consecuencias, que han señalado quienes participaron en la investigación.

Es importante señalar que la afectación en la salud de miles de personas por la aplicación del nemagón, afectación sufrida por trabajadores y trabajadoras de las plantaciones y las empacadoras bananeras, por las esposas y compañeras de los trabajadores y por los hijos e hijas de éstos, es una afectación que difícilmente se puede representar con números, ya sea para saber cuánta es la población afectada, ya sea para ponderar la afectación en términos económicos. Es así como, en este momento se desconoce cuánta es la población afectada por el nemagón, a pesar de que algunos análisis han estimado que han sido afectadas por 
este agroquímico alrededor de 30 mil personas en el país (Boix, 2007; Gómez, 2010).

En el marco de estas afectaciones, desde los años 80, pero fundamentalmente a partir de la década de los 90 , surgió un movimiento social en la búsqueda de una indemnización por parte de las entidades implicadas en el proceso de venta y distribución, o en el proceso de regulación en el uso de dicho agroquímico, que tuvo como principal consecuencia la aprobación de la Ley de Determinación de beneficios sociales y económicos para la población afectada por el DBCP, en el año 2001 (Asamblea Legislativa, 2001). Esta legislación implicó que el Estado costarricense fuese considerado como el principal responsable de la afectación por el nemagón, con lo cual, el Estado, a través de la Caja Costarricense de Seguro Social (CCSS) y del Instituto Nacional de Seguros (INS), se hizo cargo del proceso de indemnización económica de la población afectada por el agroquímico.

Es importante señalar que este proceso de indemnización no estuvo exento de conflictos, dado que, en diferentes ocasiones, diversos grupos de trabajadores y trabajadoras bananeras afectadas por el nemagón tuvieron que presentarse frente a las instalaciones del INS, a fin de solicitar que se cumpliera lo estipulado en la ley (Mora, 2013).

Considerando los elementos expuestos, es importante señalar que la afectación en la salud, en la vida familiar, en la vida cotidiana, en la vida laboral y también en las subjetividades políticas de quienes se vieron expuestos al nemagón, son procesos que pueden ser comprendidos bajo el concepto de sufrimiento ambiental. Recupero este concepto que autores como Auyero y Swistun (2008) han creado para comprender la dinámica surgida en Villa Inflamable, una villa ubicada en Buenos Aires, Argentina, y que se encuentra rodeada por polos petroquímicos, ríos altamente contaminados por desechos tóxicos, debido a la existencia de uno de los incineradores más grandes del país y un relleno sanitario, ubicación geográfica que ha generado una serie de dinámicas que expresan este fenómeno. De acuerdo a los autores, el estudio del sufrimiento ambiental

"es (junto con una indagación sobre los "datos duros" de la contaminación) un examen de las experiencias y los sentidos atribuidos a este sufrimiento. Una etnografía del sufrimiento ambiental es un análisis de las voces de quienes padecen, pero es también un estudio de las narrativas que circulan alrededor de las vidas de quienes lo padecen, esto es, de todos los intentos de darle sentido a ese sufrimiento, de todas las apropiaciones y reconocimientos que son, como lo implica el análisis, actos profundamente políticos" (Auyero y Swistun, 2008: 218).

El concepto de sufrimiento ambiental es un concepto central para comprender los procesos de afectación que han surgido en torno a la aplicación y la exposición al nemagón por parte de diversos grupos. Con este concepto es posible unificar en la discusión y en las experiencias del trabajo de investigación realizado con las personas afectadas por el agroquímico, la profunda escisión que continúa existiendo entre ambiente y cuerpo, dado que aquello que le ocurre al ambiente es algo que también le ocurre a los distintos grupos humanos, por lo que, en sus cuerpos se expresan los resultados de las dinámicas productivas, de los procesos extractivos y de las lógicas de explotación y contaminación de los recursos a los que se ha encontrado históricamente sometida, en este caso, la región del Caribe de nuestro país.

Considerando lo anterior, desde la perspectiva de las ciencias sociales, el estudio del movimiento de las trabajadoras y los trabajadores bananeros afectados por el nemagón nos pone en alerta respecto a distintos procesos que se enuncian seguidamente. La consideración de estos procesos se enmarca en las discusiones en torno a los ejes centrales que articulan el intercambio y la retroalimentación en el programa de investigación en el que se circunscribe este proyecto, cuáles son, las dinámicas de acumulación, distribución y desigualdad que se encuentran presentes en la región.

Una síntesis de estos procesos se muestra en la figura 1, que se expone a continuación. 
1. Cómo determinados actores y grupos sociales continúan poniendo "el cuerpo" a los procesos productivos y extractivos de acumulación.

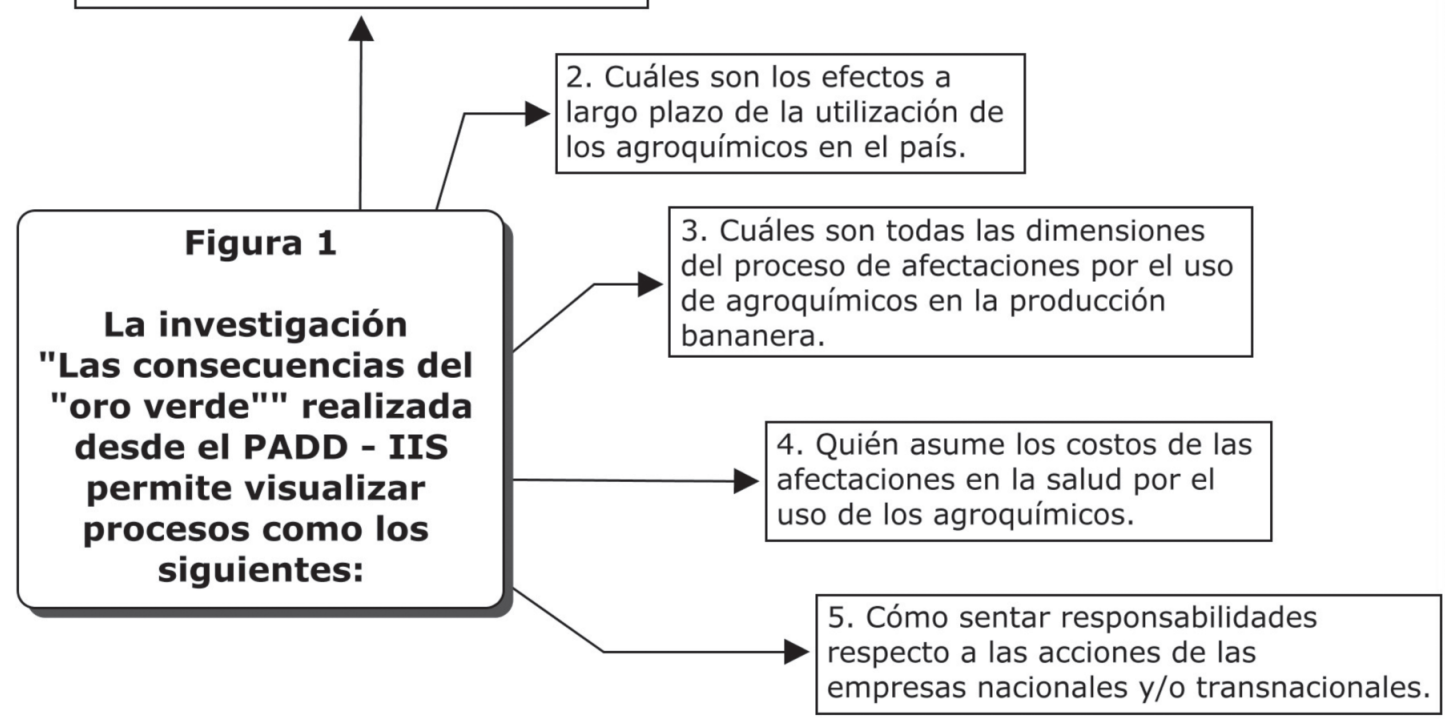

En primer lugar, el movimiento social analizado muestra cómo determinados actores y grupos sociales continúan poniendo "el cuerpo" a los procesos productivos y extractivos de acumulación. Si bien desconocemos cuántas fueron las personas afectadas por el nemagón, como se señalaba anteriormente, algunas investigaciones estiman que en Costa Rica fueron afectadas 30 mil personas por el uso de este agroquímico, cifra que incluiría a las personas afectadas directa e indirectamente. El objeto de estudio del proyecto de investigación, como se señala más adelante, es sólo una de las expresiones que se puede observar respecto a las consecuencias de procesos extractivos y productivos, de los cuales, abundan casos en la región centroamericana, y latinoamericana, también.

En segundo lugar, el proceso de investigación realizado desde el Instituto de Investigaciones Sociales (IIS) brinda señales de alerta para discutir en torno a cuáles son los efectos a largo plazo del uso de los agroquímicos en el país. Si bien el nemagón se utilizó durante los años 60 y 70 en Costa Rica, es actualmente cuando el país está empezando a comprender todas las dimensiones del proceso de afectación de la población trabajadora de las bananeras. En ese sentido, Hazel Díaz, funcionaria de la Defensoría de los Habitantes señalaba, en una mesa redonda realizada por el IIS en la sede Regional de Limón, en el año 2009, que si bien hasta ahora el Estado costarricense se encuentra resolviendo un problema que inició aproximadamente hace 50 años, algunas preguntas que tenemos pendientes en cuanto a la discusión pública del uso de los agroquímicos en el país, son cuáles van a ser las consecuencias y cómo vamos a enfrentar los procesos de afectación de agroquímicos tan letales como el bromacil, agroquímico utilizado en las plantaciones piñeras (Arguedas; Boeglin; Díaz, 2009). En ese sentido, considerar que el uso de agroquímicos es una de las características fundamentales de la producción de monocultivos, y que por lo tanto, el uso del nemagón no es una experiencia aislada, por lo que pueden surgir eventuales afectaciones de este tipo a raíz de otros agroquímicos, es esencial para atender las consecuencias del uso de nuevos agroquímicos en la producción de monocultivos a nivel nacional. 
Una tercera artista a considerar refiere a la necesidad de profundizar en cuáles son todas las dimensiones del proceso de afectación por el uso de agroquímicos en la producción bananera. El proceso de afectación en la salud de los trabajadores bananeros se entendió, por parte de la organización política y sindical, por parte de los medios de comunicación y por parte de los abogados y de algunos médicos que se involucraron en el proceso, como un problema de esterilidad masculina. Sin embargo, la realización de entrevistas en el marco del proyecto muestra a hombres hablando no sólo de su esterilidad, sino también de la ruptura en las relaciones familiares, y de lo que ellos denominan "el abandono" o "el cambio" que las mujeres han hecho de los hombres afectados por el nemagón, buscando hombres "no enfermos". De la misma manera, los hombres han hablado de los procesos de estigmatización de los trabajadores, en donde éstos son llamados en sus comunidades y por sus familiares "los capones" o "los esterilizados". De igual forma, al encontrarse la lucha enfocada en torno al reconocimiento de la esterilidad masculina, se dejaron de lado los padecimientos de las mujeres, independientemente de que éstas fueran trabajadoras bananeras, y de los hijos e hijas de estos trabajadores. A esto se unen los relatos en torno a los constantes dolores de cabeza, los dolores en las articulaciones, los problemas respiratorios, de enfermedades en la piel, que por lo general, no fueron reconocidos o no fueron articulados claramente como demandas dentro del movimiento social (Mora, 2013). Es por ello, que intentar profundizar en todas las dimensiones que tiene el proceso de afectación del uso de los agroquímicos es un proceso central de las reflexiones, los debates y los aportes que se pueden realizar desde las ciencias sociales.

En cuarto lugar, es fundamental preguntarse quién asume los costos de las afectaciones en la salud por el uso de los agroquímicos. La pregunta que surge es por qué debe ser el Estado costarricense y las instituciones públicas, como la Caja Costarricense de Seguro Social (CCSS) y el Instituto Nacional de Seguros (INS), las entidades que asuman los riesgos y las consecuencias en la salud que surgen como resultado de procesos productivos y extractivos de carácter privado. Es claro que esta pregunta no viene a señalar que la sociedad costarricense debe negarles servicios públicos y de calidad a las personas que se han visto afectadas por procesos productivos y extractivos. Lo que viene a señalar este cuestionamiento es que el Estado costarricense sigue siendo un Estado que "paga los platos rotos" de los procesos de acumulación, extracción y expropiación de recursos que se viven en el país, siendo las personas trabajadoras y las comunidades las que siguen poniendo el cuerpo y aportando su cuota de sufrimiento ambiental, tal y como se señalaba anteriormente, quedando las empresas nacionales y transnacionales impunes, y bajo el resguardo garantizado por la institucionalidad costarricense.

Finalmente, desde esta experiencia de investigación un quinto proceso que es central problematizar es el cómo sentar responsabilidades respecto a las acciones y consecuencias del accionar de las empresas nacionales y/o transnacionales. Quizá este sea uno de los ejes más difíciles de problematizar en temas como el propuesto, dado que las empresas nacionales y transnacionales parecen ser uno de los actores más difíciles de hacer responder en cuanto a procesos de afectación en la salud o en el resarcir de daños al ambiente. Pensar en torno a estas responsabilidades nos lleva a reflexionar alrededor de los mecanismos para discutir cómo podemos poner a responder a sujetos jurídicos, más allá de la censura moral y más allá de lo que se puede lograr desde el repertorio de la protesta social, sin que la factura de los procesos de acumulación le sea endosada al ámbito público, mientras se sigue acumulando riqueza en el ámbito privado.

\section{¿Cuál ciencia social?}

El trabajo de investigación realizado desde PADD en el Instituto de Investigaciones Sociales (IIS) permite reflexionar en torno al quehacer de las ciencias sociales a partir de esta experiencia de investigación, por lo que, lo que seguidamente se expone es el resultado de la reflexión que acompaña la ejecución del proyecto ya citado. A partir de esta experiencia en particular, algunas ideas respecto a cómo podemos imaginar las ciencias sociales, sus retos y sus posibilidades son las que aparecen sistematizadas en la figura 2 . 


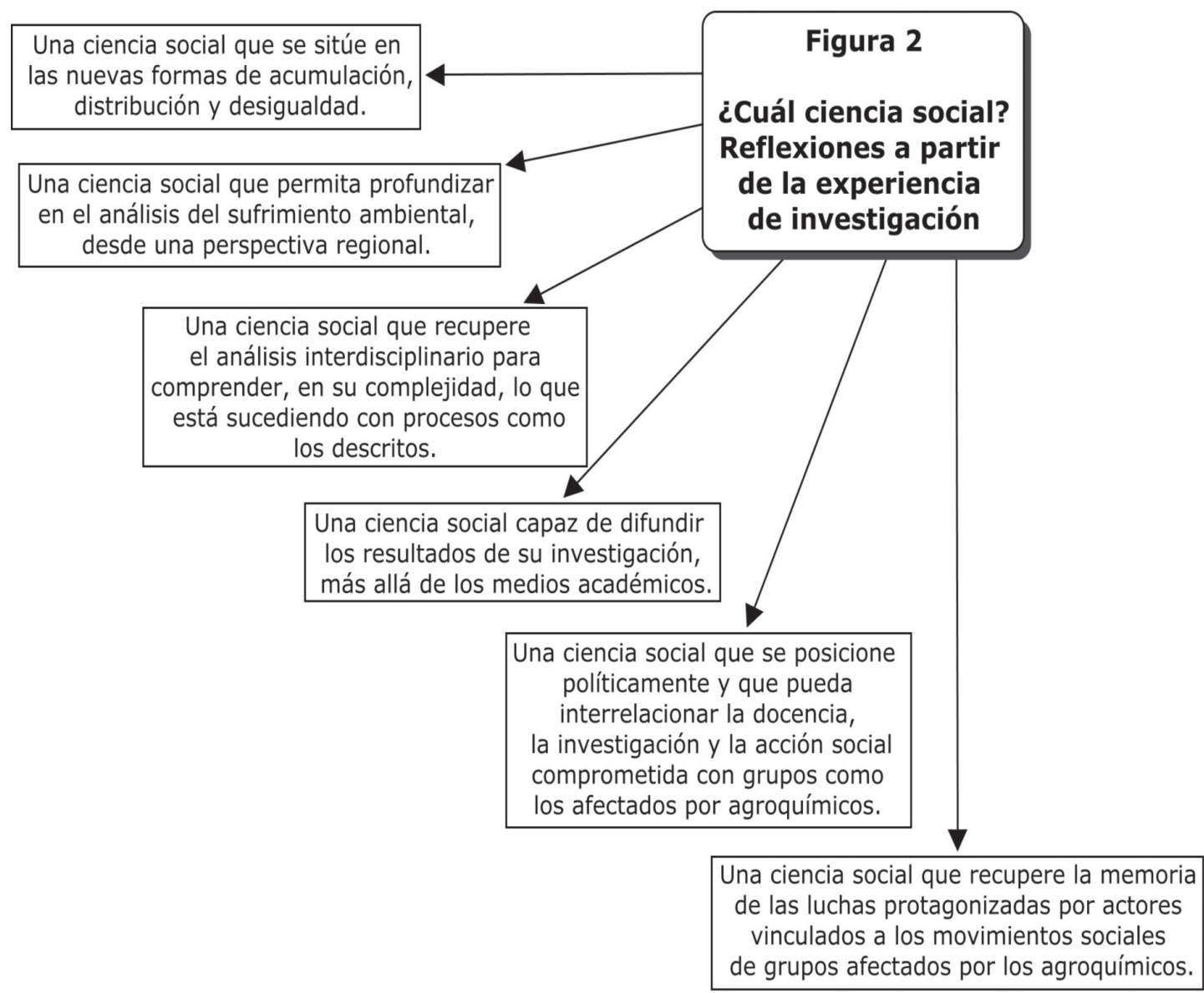

A partir de la experiencia de investigación citada, se vuelve necesaria una ciencia social que se sitúe en las nuevas formas de acumulación, distribución y desigualdad. La experiencia de investigación descrita se sitúa en un programa de investigación en donde el énfasis del análisis refiere, justamente, a los procesos señalados. Es por ello que si bien en otro momento histórico el tema de los agroquímicos poco tenía que ver con las ciencias sociales, hoy en día, el uso de agroquímicos, sus efectos en la salud de las personas, en las relaciones laborales, en las vidas familiares y en las subjetividades políticas invitan a pensar en el aporte que podemos dar desde las ciencias sociales al entendimiento de esas nuevas formas de acumulación de la riqueza y de distribución de los efectos y las consecuencias de la contaminación, de los riesgos, las enfermedades y el sufrimiento, en términos generales.

De la misma manera, y en segundo lugar, esta es una experiencia que invita a profundizar en una ciencia social, y en el análisis del sufrimiento ambiental, desde una perspectiva regional. Desde las ciencias sociales, es necesario comprender qué es lo que está sucediendo en las distintas regiones del país, en términos de las dinámicas que surgen con las plantaciones bananeras, con las plantaciones piñeras, con las plantaciones de caña, de melón y las flores, y en las expresiones de determinadas formas de sufrimiento ambiental en estas regiones del país. De la misma forma, distintas investigaciones muestran cómo lo que está sucediendo en las distintas regiones de Costa Rica, no es distinto a lo que sucede en otros países a nivel centroamericano, 
como por ejemplo lo muestran las consecuencias de la siembra de caña de azúcar para el caso de Nicaragua (Cuadra; Jakobsson; Hogstedt, Wesseling, 2006), la contaminación con arsénico producto de la actividad minera en Honduras y en Guatemala (Ravenga, 2011), o a nivel latinoamericano, la siembra de flores en Colombia (Harari, 2004; Korovkin, 2004) o la soja en Argentina (Rulli, 2009).

En tercer lugar, es fundamental propiciar una ciencia social que permita profundizar en el análisis interdisciplinario para comprender, en su complejidad, qué es lo que está sucediendo en procesos como los descritos. La ejecución del proyecto de investigación ha mostrado cuán necesarios son los equipos interdisciplinarios en la realización de un trabajo de investigación de carácter etnográfico como el señalado. Es claro que esta "necesidad" toca directamente el tema de la disponibilidad de los recursos para investigar, tema que no está de más plantear en el marco de unas Jornadas de Reflexión en torno a las ciencias sociales. Aunado al tema de los recursos, resulta fundamental traer a esta discusión que la interdisciplinariedad propuesta a partir de la investigación realizada involucra tanto a las disciplinas de las ciencias sociales, como a las disciplinas de otras áreas, como lo pueden ser, el trabajo directo con los profesionales en medicina, con los profesionales en salud pública y con los abogados. Sin duda, la investigación del movimiento del nemagón y el trabajo de campo realizado apuntan a la necesidad de trabajar en ese sentido, considerando también que de acuerdo a las voces de los afectados por el nemagón, muchos abogados se han acercado al movimiento y diversas organizaciones han surgido, no con el interés de ayudar a quienes se vieron afectados, sino con la intención de obtener ganancias económicas, lucrando a costa de su sufrimiento ambiental (Mora, 2013).

De la misma manera, resulta necesaria una ciencia social capaz de difundir los resultados de su investigación más allá de los medios académicos. La necesidad de divulgar los resultados de investigación es fundamental más allá de los mecanismos y recursos con los que ya contamos, como las conferencias públicas o las revistas especializadas que fortalecen la vida académica. Si bien la experiencia de investigación descrita tiene un blog que cuenta al mes de julio con 7212 visitas (el blog se encuentra disponible en la dirección http://consecuenciasdeloroverde.blogspot.com/), a todas luces resulta insuficiente para dar a conocer la experiencia de investigación, y mucho más, es insuficiente si se trata de pensar la investigación con alguna incidencia política.

Adicionalmente, es necesaria una ciencia social que profundice en el análisis político y que pueda interrelacionar la docencia, la investigación y la acción social comprometida con grupos como estos. Si bien se han planteado algunos temas que tienen que ver con resultados del proceso de investigación, imaginar procesos de acompañamiento, desde la acción social y desde la docencia, de los grupos sociales y actores que se encuentran en procesos de sufrimiento ambiental es fundamental para que estos trabajos puedan tener una mayor incidencia política.

Finalmente, es fundamental y necesaria una ciencia social que recupere la memoria de las luchas protagonizadas por actores vinculados a los movimientos sociales de grupos afectados por los agroquímicos, que ayude a comprender los procesos de autoconstitución y configuración política, para no dejar en el olvido las luchas y las resistencias contra los procesos de acumulación, distribución y desigualdad.

\section{Conclusiones}

El tema del uso y exposición a los agroquímicos en el contexto de expansión de los monocultivos tiene una amplia relación con las ciencias sociales, si las consecuencias desarrolladas ante estas actividades se inscriben y se analizan en el marco de las lógicas y los mecanismos de acumulación, distribución y desigualdad, que caracterizan los procesos como los descritos. De la misma manera, el uso y la exposición a los agroquímicos tienen una gran relación con las ciencias sociales, en términos de las reflexiones y los aportes que se pueden realizar desde las especificidades, pero también, desde las interrelaciones y desde el intercambio de las distintas tareas realizadas a partir de la investigación, la docencia y la acción social. 
La preocupación en torno a qué hacemos con aquello que investigamos es una pregunta central que fue planteada en muchas de las mesas presentadas en el marco de las Jornadas de Reflexión de Ciencias Sociales. En ese sentido, la memoria, la indagación, la denuncia y el acompañamiento que desde las ciencias sociales se puede brindar a grupos sociales y actores que viven en condiciones de sufrimiento ambiental es parte central de los retos pendientes para nuestras disciplinas.

\section{Referencia bibliógrafica}

Arguedas, C; Boeglin, N; Hazel, D. (2009). Mesa redonda "Del nemagón al bromacil: monocultivos, luchas sociales y ambiente". Limón: Instituto de Investigaciones Sociales (IIS) - Sede Regional de Limón de la Universidad de Costa Rica (UCR). 22 de setiembre de 2009.

Asamblea Legislativa de Costa Rica. (2001). Ley de Determinación de beneficios sociales y económicos para la población afectada por el DBCP. Asamblea Legislativa. Costa Rica.

Auyero, J; Swistun, D. (2008). Inflamable. Estudio del sufrimiento ambiental. Paidós Editorial. Argentina. $240 \mathrm{p}$.

Barraza, D; Jansen, K; van Wendel, B; Wesswling, C. (2013). Social movement and risk perception: unions, churches, pesticides and bananas in Costa Rica. International Journal of Occupational and Environmental Health. Número 19. 11-21.

Bohmen, S. (2012). The Role of the Nation-State in the Transnational History of DBCP. Sellers, C; Mellig, J. (editores). Dangerous Trade. Histories of Industrial Hazard across a globalizing World. Temple University Press. Philadelphia. 168-180.

Boix, V. (2007). El parque de las hamacas. El químico que golpeó a los pobres. Icaria Editorial. España. 318 p.

Cuadra, S; Jakobsson, K; Hogstedt,C; Wesseling, C. (2006). Enfermedad Renal Crónica: Evaluación del conocimiento actual y la viabilidad para la colaboración de su investigación a nivel regional en América
Central. Serie Salud y Trabajo, No 2 . SALTRA, IRET-UNA. Costa Rica. 76 p.

Giralt, M. (1996). La problemática ética del uso del DBCP en Costa Rica. Revista de Filosofía. Universidad de Costa Rica. XXXIV. 83-84. 415-421.

Gómez, C. (2009). Lucha social y organización bananera: el caso de las afectaciones en extrabajadores en Chinandega, Nicaragua. Tesis para optar por el grado de Doctora. Centro de Estudios Rurales del Colegio de Michoacán. México. 290 p.

Harari, R. (2004). La economía de exportación y la salud. Los casos de petróleo, banano y flores. Korovkin, T. (compiladora). Efectos sociales de la globalización: petróleo, banano y flores en Ecuador. Centro de Investigación de los Movimientos Sociales del Ecuador (CEDIME). Ecuador. 185-227.

Korovkin, T. (2004). Globalización y pobreza: los efectos sociales del desarrollo de la floricultura de exportación. Korovkin, T. (compiladora). Efectos sociales de la globalización: petróleo, banano y flores en Ecuador. Centro de Investigación de los Movimientos Sociales del Ecuador (CEDIME). Ecuador. 79-127.

Mora, S. (2011). Blog del proyecto Consecuencias del Oro Verde. Disponible en la dirección http://consecuenciasdeloroverde.blogspot. com.

Mora, S. (2013). Reflexiones para el análisis comparativo de movimientos sociales: el caso de las extrabajadoras y extrabajadores bananeros afectados por el nemagón en Costa Rica y Nicaragua. Anuario de Estudios Centroamericanos. Número 39. En prensa.

Ravenga, Á. (2011). El oro o la vida. Recolonización y resistencia en Centroamérica. Caracol Producciones. Guatemala.

Rulli, J. (2009). Pueblos fumigados. Los efectos de los plaguicidas en las regiones sojeras. Editorial del Nuevo Extremo. Argentina. $400 \mathrm{p}$. 